\title{
Tight-binding dispersion of the prismatic pentagonal lattice
}

\author{
Susobhan Paul* \\ Department of Physics, Scottish Church College, \\ Urquhart Square, Kolkata 700006, India \\ Asim Kumar Ghosh ${ }^{\dagger}$ \\ Department of Physics, Jadavpur University, \\ 188 Raja Subodh Chandra Mallik Road, Kolkata 700032, India
}

\begin{abstract}
Tight-binding Hamiltonian on the prismatic pentagonal lattice is exactly solved to obtain the analytic expressions of dispersion relations and eigenvectors. This lattice is made of prismatic pentagon which is different from Cairo pentagon. Six different dispersion relations and total density of states are obtained. Dispersion relations are symmetric about the zero energy at a particular point in the parameter space. Although a large gap is found for the Cairo pentagonal lattice, no gap as well as no Dirac cone is found to appear in the tight-binding band structure for this prismatic pentagonal lattice. Instead, a pair of van Hove singularities has been identified at two different energy values in the band structure.
\end{abstract}

*Electronic address: suso.phy.paul@gmail.com

†Electronic address: asimkumar96@yahoo.com 


\section{INTRODUCTION}

Investigations on electronic properties of two-dimensional (2D) models exhibit significant growth after the successful realization of graphene, a 2D honeycomb structure of carbons. One particular choice of unit cell for honeycomb lattice is a hexagon. Although the regular polygons like triangle, square and hexagon could fill up a 2D planar surface without overlap and leaving any voids, it is impossible to do so by using the regular pentagons due to its five-fold symmetry. Thus to fill up a planar surface one has to use irregular pentagons those are derived from the regular one by changing the lengths of the arms as well as the angles between them. The examples of irregular pentagons those can fill planar surface are prismatic and Cairo pentagons. Structures of prismatic and Cairo pentagons are shown in Fig. 1 (b) and (e), respectively. Electronic properties of the Cairo pentagonal lattice have been derived through a number of theoretical studies in more recent times. Those results confirm the presence of a large gap in its band structure. Magnitude of this gap is $3.25 \mathrm{eV}$ which is estimated by density functional theory (DFT) on a particular system that is known as pentagraphene (p-graphene). P-graphene is essentially a Cairo pentagonal monolayer of carbon atoms [1]. DFT analysis on other Cairo pentagonal monolayers composed of boron nitride and silver azide indicate the presence of a single band-gap as well [2]. The tight-binding (TB) analysis on p-graphene supports the existence of the same single band-gap whose estimated value is very close to the previous prediction [3]. It has been proposed that a stable carbon allotrope resembling p-graphene could be materialized very soon which is entirely composed of Cairo-typed carbon pentagons [1]. Besides those electronic properties, magnetic properties of frustrated antiferromagnetic (AFM) compounds, $\mathrm{Bi}_{2} \mathrm{Fe}_{4} \mathrm{O}_{9}$ [4], $\mathrm{Bi}_{4} \mathrm{Fe}_{5} \mathrm{O}_{1} 3 \mathrm{~F}$ [5] and organic radical crystal, $\alpha-2,6-\mathrm{Cl}_{2}-\mathrm{V}$ [= $\alpha$-3-(2,6-dichlorophenyl)-1,5-diphenylverdazyl $]$ [6] have been reported on the basis of Cairo pentagonal lattice structure. An extensive theoretical investigation of magnetic properties on AFM Heisenberg Cairo pentagonal lattice could be found in the article [7]. Ground state phase diagram and magnetization process of the spin-1/2 AFM Heisenberg Cairo pentagonal lattice in the presence of external magnetic field are available in [8] and [9], respectively.

On the other hand, very few theoretical results are available for 2D lattice composed of prismatic pentagons. The bond-percolation threshold is determined and the stability of collinear Néel-type classical ground state for AFM Heisenberg system on this lattice is 
studied. In addition, ground state entropy and energy are estimated for the Ising and AFM Heisenberg models, respectively [10, 11]. But, the existence of AFM compounds based on prismatic pentagons are not reported so far. Similarly, electronic properties of 2D prismatic pentagonal lattice remain unexplored too.

In this study, we introduce a TB model on the pentagonal lattice that is entirely composed of prismatic pentagons (Fig. 1 (a)). The lattice could be derived from the honeycomb lattice in the following way. The parent (honeycomb) lattice is elongated vertically in such a manner that vertical bonds get doubled in its original length. Additional lattice points are introduced in the middle of each vertical bonds. After joining these additional points this particular structure of pentagonal lattice is obtained. In the resulting lattice, any horizontal layer is made of prismatic pentagons by placing them side by side without overlapping and leaving any voids. The adjacent layers those are just vertically above and below of this one are made of inverted pentagons. The lattice is non-Bravais. One may visualize this lattice as composed of six interpenetrating rectangular Bravais lattices, those are identical to each other. In Fig. 1 (a), these sublattices are identified by $\mathrm{B}_{1}, \mathrm{~B}_{2}, \mathrm{~B}_{3}, \mathrm{C}_{1}, \mathrm{C}_{2}$ and $\mathrm{C}_{3}$. Those sublattices can be divided into two types on the basis of their coordination numbers. Coordination numbers of both $\mathrm{B}_{1}$ and $\mathrm{C}_{1}$ are four while they are three for the others. The rectangular unit cell of this lattice is shown in Fig. 1 (c). It effectively contains one lattice point for each of six different sublattices. This lattice has the translational invariance of length $\sqrt{3} a$ along the $x$-direction and that of length $5 a$ along the $y$-direction, where $a$ is the length of shorter arms of the pentagon. It also has rotational invariance of the angle $180^{\circ}$. The first Brillouin zone is shown in Fig. 1 (d).

In this investigation, we have derived the analytic expressions of six different dispersion relations exactly along with total density of states (DOS) for the prismatic pentagonal lattice. The results indicate no gap in the band structure for prismatic pentagonal lattice in contrast to the case of Cairo pentagonal lattice. In addition, a pair of van Hove singularities (VHS) is found to appear at two different energy values in the TB band structure of this lattice. Therefore, it is expected that electronic properties of carbon allotrope based on the prismatic pentagonal lattice would be different from that of the Cairo pentagonal lattice. The TB model and its exact solution are presented in the section II while the section III contains discussion on the results. 


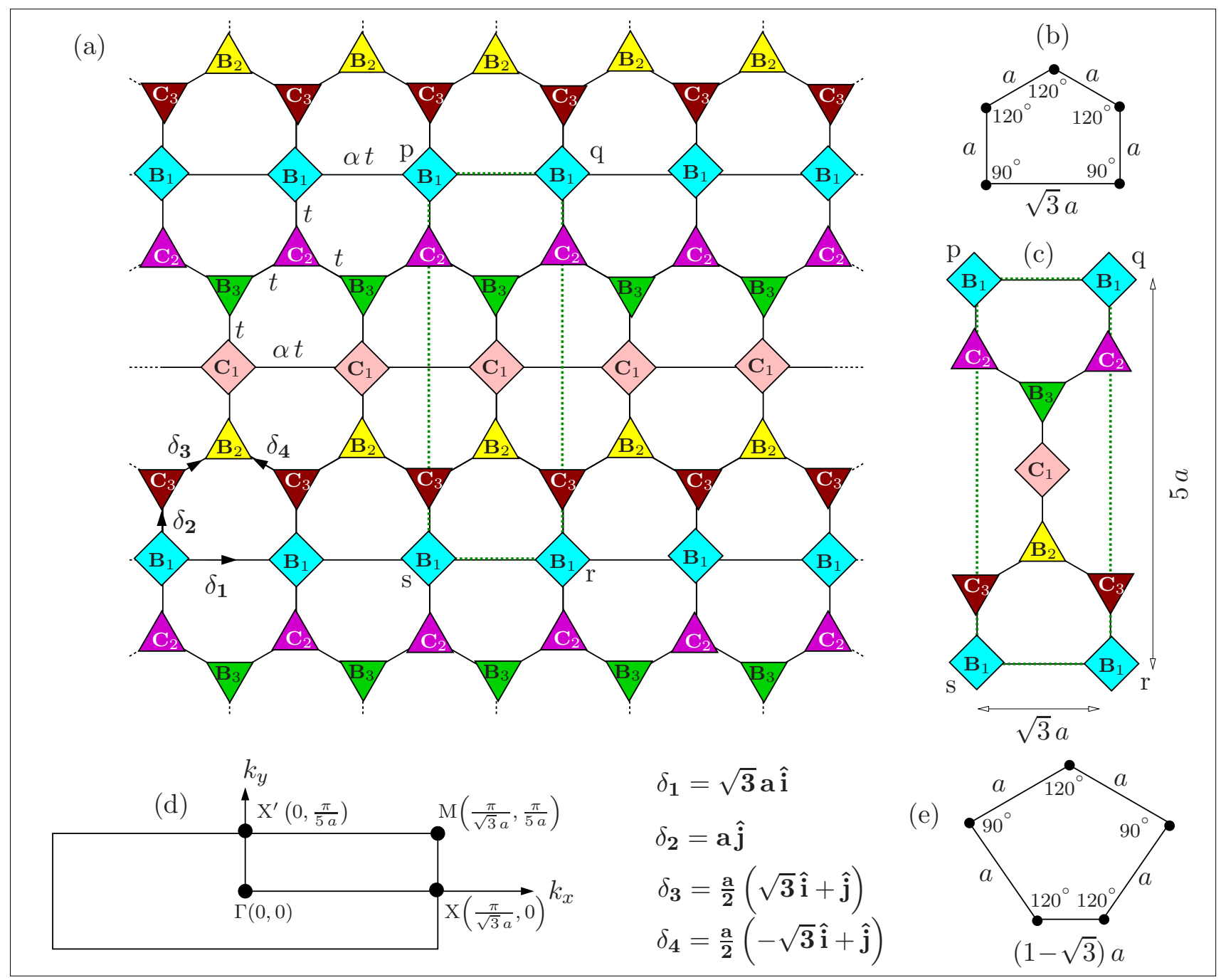

FIG. 1: (Color online) (a) pentagonal lattice, (b) prismatic pentagon, (c) unit cell, (d) first Brillouin zone and (e) Cairo pentagon. 


\section{THE TIGHT-BINDING HAMILTONIAN AND DISPERSION RELATIONS}

Considering only nearest-neighbor (NN) hopping, the model is described by the tightbinding Hamiltonian,

$$
\begin{aligned}
& H=-t \sum_{i}\left[b_{1, \mathbf{R}_{\mathbf{i}}}^{\dagger} c_{3, \mathbf{R}_{\mathbf{i}}+\delta_{\mathbf{2}}}+\alpha b_{1, \mathbf{R}_{\mathbf{i}}}^{\dagger} b_{1, \mathbf{R}_{\mathbf{i}}+\delta_{\mathbf{1}}}+b_{1, \mathbf{R}_{\mathbf{i}}}^{\dagger} c_{2, \mathbf{R}_{\mathbf{i}}-\delta_{\mathbf{2}}}+c_{2, \mathbf{R}_{\mathbf{i}}+\mathbf{3} \delta_{\mathbf{2}}+\delta_{\mathbf{3}}+\delta_{\mathbf{4}}}^{\dagger} b_{1, \mathbf{R}_{\mathbf{i}}+\mathbf{4} \delta_{\mathbf{2}}+\delta_{\mathbf{3}}+\delta_{\mathbf{4}}}\right. \\
& +c_{2, \mathbf{R}_{\mathbf{i}}+\mathbf{3} \delta_{\mathbf{2}}+\delta_{\mathbf{3}}+\delta_{\mathbf{4}}}^{\dagger} b_{3, \mathbf{R}_{\mathbf{i}}+\mathbf{3} \delta_{\mathbf{2}}+\delta_{\mathbf{3}}}+c_{2, \mathbf{R}_{\mathbf{i}}+\mathbf{3} \delta_{\mathbf{2}}+\delta_{\mathbf{3}}+\delta_{\mathbf{4}}}^{\dagger} b_{3, \mathbf{R}_{\mathbf{i}}+\mathbf{3} \delta_{\mathbf{2}}+\delta_{\mathbf{4}}}+b_{3, \mathbf{R}_{\mathbf{i}}+\mathbf{3} \delta_{\mathbf{2}}+\delta_{\mathbf{3}}}^{\dagger} c_{1, \mathbf{R}_{\mathbf{i}}+\mathbf{2} \delta_{\mathbf{2}}+\delta_{\mathbf{3}}} \\
& +b_{3, \mathbf{R}_{\mathbf{i}}+\mathbf{3} \delta_{\mathbf{2}}+\delta_{\mathbf{3}}}^{\dagger} c_{2, \mathbf{R}_{\mathbf{i}}+\mathbf{3} \delta_{\mathbf{2}}+\mathbf{2} \delta_{\mathbf{3}}}+b_{3, \mathbf{R}_{\mathbf{i}}+\mathbf{3} \delta_{\mathbf{2}}+\delta_{\mathbf{3}}}^{\dagger} c_{2, \mathbf{R}_{\mathbf{i}}+\mathbf{3} \delta_{\mathbf{2}}+\delta_{\mathbf{3}}+\delta_{\mathbf{4}}}+c_{1, \mathbf{R}_{\mathbf{i}}+\mathbf{2} \delta_{\mathbf{2}}+\delta_{\mathbf{3}}}^{\dagger} b_{2, \mathbf{R}_{\mathbf{i}}+\delta_{\mathbf{2}}+\delta_{\mathbf{3}}} \\
& +c_{1, \mathbf{R}_{\mathbf{i}}+\mathbf{2} \delta_{\mathbf{2}}+\delta_{\mathbf{3}}}^{\dagger} b_{3, \mathbf{R}_{\mathbf{i}}+\mathbf{3} \delta_{\mathbf{2}}+\delta_{\mathbf{3}}}+\alpha c_{1, \mathbf{R}_{\mathbf{i}}+\mathbf{2} \delta_{\mathbf{2}}+\delta_{\mathbf{3}}}^{\dagger} c_{1, \mathbf{R}_{\mathbf{i}}+\delta_{\mathbf{1}}+\mathbf{2} \delta_{\mathbf{2}}+\delta_{\mathbf{3}}}+b_{2, \mathbf{R}_{\mathbf{i}}+\delta_{\mathbf{2}}+\delta_{\mathbf{3}}}^{\dagger} c_{1, \mathbf{R}_{\mathbf{i}}+\mathbf{2} \delta_{\mathbf{2}}+\delta_{\mathbf{3}}}
\end{aligned}
$$

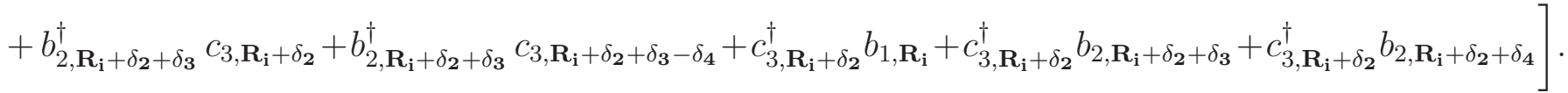

$t$ and $\alpha t$ are the hopping parameters across the NN sites having the bonds of lengths $a$ and

$\sqrt{3} a$, respectively (Fig.1 (a)). $\mathbf{R}_{\mathbf{i}}$ is the Bravais vector for the $i$-th unit cell. $b_{j}^{\dagger}\left(c_{j}^{\dagger}\right)$ is the creation operator at the $j$-th site with respective orbital for an atom on the B (C) sublattice. $\delta_{\mathrm{i}} \mathrm{s}$ are the vectors pointing different adjacent lattice sites within each unit cell. Hopping parameters are generally depend on the bond lengths in the system. By using the hopping parameter-bond length relationship introduced by Harrison, i. e., $t(a) \propto 1 / a^{2}$, value of $\alpha$ is determined, and that is equal to $1 / 3$ [12]. This kind of parametrization for the AFM exchange interaction strengths has been adopted before while investigating the stability of collinear Néel-type classical ground state of Heisenberg Hamiltonian on this pentagonal lattice. Interestingly, in that case, the critical value of $\alpha, i$. e., $\alpha_{c}$ is also very close to $1 / 3$ above which the classical ground state is no longer stable [11]. We have considered four closer points to each of $\mathrm{B}_{1}$ and $\mathrm{C}_{1}$ sub-lattice sites as the $\mathrm{NN}$ points despite their different bond lengths. For other sites, number of NN points are three and their arrangements are like the honeycomb lattice. The orbitals are considered to be the simplest one like the $\pi$ electron of graphene for the sites $\mathrm{B}_{2}, \mathrm{~B}_{3}, \mathrm{C}_{2}$ and $\mathrm{C}_{3}$. However, no specific orbital structure is assumed for the $\mathrm{B}_{1}$ and $\mathrm{C}_{1}$ lattice sites.

In order to obtain dispersion relations, the Hamiltonian is expressed in $\mathbf{k}$-space by transforming the $b$ and $c$ operators as

$$
b_{n \mathbf{k}}=\frac{1}{\sqrt{N}} b_{n, \mathbf{R}_{\mathbf{i}}} e^{i \mathbf{k} \cdot \mathbf{R}_{\mathbf{i}}}, \quad c_{n \mathbf{k}}=\frac{1}{\sqrt{N}} c_{n, \mathbf{R}_{\mathbf{i}}} e^{i \mathbf{k} \cdot \mathbf{R}_{\mathbf{i}}}, \quad n=1,2,3,
$$

where $N$ is the total number of unit cells in the lattice. By introducing the six component 
vector,

$$
\Psi_{\mathbf{k}}^{\dagger}=\left(\begin{array}{llllll}
b_{1 \mathbf{k}}^{\dagger} & b_{2 \mathbf{k}}^{\dagger} & b_{3 \mathbf{k}}^{\dagger} & c_{1 \mathbf{k}}^{\dagger} & c_{2 \mathbf{k}}^{\dagger} & c_{3 \mathbf{k}}^{\dagger}
\end{array}\right),
$$

$H$ can be written as,

$$
H=-t \sum_{\mathbf{k}} \Psi_{\mathbf{k}}^{\dagger} H_{\mathbf{k}} \Psi_{\mathbf{k}},
$$

where the $6 \times 6$ Hamiltonian in the momentum space is decomposed in terms of two $3 \times 3$ block Hamiltonians, $\Omega_{1}$ and $\Omega_{2}$. Therefore,

$$
\begin{aligned}
H_{\mathbf{k}} & =\left(\begin{array}{ll}
\Omega_{1} & \Omega_{2} \\
\Omega_{2} & \Omega_{1}
\end{array}\right), \quad \Omega_{1}=\left(\begin{array}{ccc}
X & 0 & 0 \\
0 & 0 & 0 \\
0 & 0 & 0
\end{array}\right), \quad \Omega_{2}=\left(\begin{array}{ccc}
0 & Y & Y \\
Y & 0 & Z \\
Y & Z & 0
\end{array}\right), \text { where } \\
X & =-\alpha t \cos \left(\sqrt{3} k_{x} a\right), \quad Y=-t \cos \left(k_{y} a\right), \quad \text { and } Z=-2 t \cos \left(\frac{k_{y} a}{2}\right) \cos \left(\frac{\sqrt{3} k_{x} a}{2}\right) .
\end{aligned}
$$

The Hamiltonian, $H_{\mathbf{k}}$ can be diagonalized by introducing the transfer matrix, $T$ such that,

$$
H_{\mathrm{k}}=\Phi_{\mathrm{k}}^{\dagger} H_{\mathrm{k}}^{d} \Phi_{\mathrm{k}}, \quad \Phi_{\mathbf{k}}=T \Psi_{\mathrm{k}},
$$

where

$$
\begin{aligned}
& H_{\mathrm{k}}^{\mathrm{d}}=\left(\begin{array}{cc}
E^{+} & 0 \\
0 & E^{-}
\end{array}\right), \quad T=\left(\begin{array}{cc}
T_{1} & T_{2} \\
T_{3} & T_{4}
\end{array}\right), \quad E^{ \pm}=\left(\begin{array}{ccc}
E_{1}^{ \pm} & 0 & 0 \\
0 & E_{2}^{ \pm} & 0 \\
0 & 0 & E_{3}^{ \pm}
\end{array}\right), \\
& T_{1}=\left(\begin{array}{ccc}
0 & \frac{2 Y}{\left(X-E_{2}^{+}\right) \sqrt{N_{+}}} & \frac{2 Y}{\left(X-E_{3}^{+}\right) \sqrt{N_{+}^{\prime}}} \\
-\frac{1}{2} & -\frac{1}{\sqrt{N_{+}}} & \frac{1}{\sqrt{N_{+}^{\prime}}} \\
\frac{1}{2} & -\frac{1}{\sqrt{N_{+}}} & \frac{1}{\sqrt{N_{+}^{\prime}}}
\end{array}\right), \quad T_{2}=\left(\begin{array}{ccc}
0 & \frac{2 Y}{\left(X-E_{2}^{-}\right) \sqrt{N_{-}}} & \frac{2 Y}{\left(X-E_{3}^{-}\right) \sqrt{N_{-}^{\prime}}} \\
\frac{1}{2} & -\frac{1}{\sqrt{N_{-}}} & \frac{1}{\sqrt{N_{-}^{\prime}}} \\
-\frac{1}{2} & -\frac{1}{\sqrt{N_{-}}} & \frac{1}{\sqrt{N_{-}^{\prime}}}
\end{array}\right) \text {, } \\
& T_{3}=\left(\begin{array}{ccc}
0 & \frac{2 Y}{\left(X-E_{2}^{+}\right) \sqrt{N_{+}}} & \frac{2 Y}{\left(X-E_{3}^{+}\right) \sqrt{N_{+}^{\prime}}} \\
-\frac{1}{2} & -\frac{1}{\sqrt{N_{+}}} & \frac{1}{\sqrt{N_{+}^{\prime}}} \\
\frac{1}{2} & -\frac{1}{\sqrt{N_{+}}} & \frac{1}{\sqrt{N_{+}^{\prime}}}
\end{array}\right), \quad T_{4}=\left(\begin{array}{ccc}
0 & \frac{2 Y}{\left(X-E_{2}^{-}\right) \sqrt{N_{-}}} & \frac{2 Y}{\left(X-E_{3}^{-}\right) \sqrt{N_{-}^{\prime}}} \\
-\frac{1}{2} & -\frac{1}{\sqrt{N_{-}}} & \frac{1}{\sqrt{N_{-}^{\prime}}} \\
\frac{1}{2} & -\frac{1}{\sqrt{N_{-}}} & \frac{1}{\sqrt{N_{-}^{\prime}}}
\end{array}\right) \text {, } \\
& N_{ \pm}=4\left(1+2\left(\frac{Y}{X-E_{2}^{ \pm}}\right)^{2}\right), \quad N_{ \pm}^{\prime}=4\left(1+2\left(\frac{Y}{X-E_{3}^{ \pm}}\right)^{2}\right) \text {. }
\end{aligned}
$$


$H_{\mathbf{k}}^{\mathrm{d}}$ is the diagonalized Hamiltonian and $\Phi_{\mathbf{k}}$ is the eigenvector. The exact expressions of six dispersion relations, $E_{n}^{ \pm}, n=1,2,3$, are written below.

$$
\begin{aligned}
& E_{1}^{ \pm}=\mp Z \\
& E_{2}^{ \pm}=\frac{1}{2}\left(X+Z \pm \sqrt{(X-Z)^{2}+8 Y^{2}}\right) \\
& E_{3}^{ \pm}=\frac{1}{2}\left(X-Z \pm \sqrt{(X+Z)^{2}+8 Y^{2}}\right) .
\end{aligned}
$$

The dispersion relations, $E_{n}^{ \pm}, n=1,2,3$ for $\alpha=1$ have been drawn in different colors within the first Brillouin zone (1BZ) and shown in Fig. 2. The DOS, $g(E)$ could be derived by using the formula,

$$
g(E)=\frac{5 \sqrt{3}}{(2 \pi)^{2}} \int_{1 \mathrm{BZ}} d \mathbf{k} \sum_{\mathbf{n}=\mathbf{1 , 2}, \mathbf{3} ; \gamma=+,-} \delta\left(\mathbf{E}-\mathbf{E}_{\mathbf{n}}^{\gamma}(\mathbf{k})\right) .
$$

Six dispersion relations, $E_{1}^{+}, E_{2}^{+}, E_{3}^{+}, E_{1}^{-}, E_{2}^{-}$and $E_{3}^{-}$along with DOS for $\alpha=0,1 / 3,2 / 3$

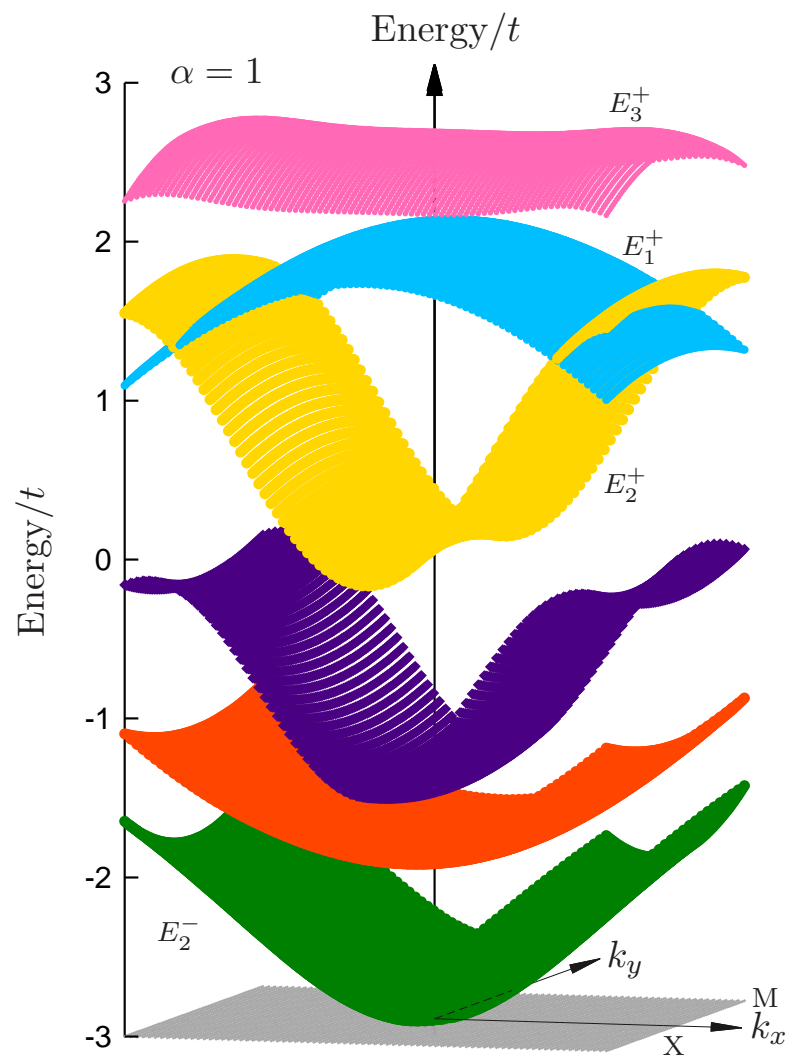

FIG. 2: (Color online) The dispersion relations, $E_{1}^{+}, E_{2}^{+}, E_{3}^{+}, E_{1}^{-}, E_{2}^{-}$and $E_{3}^{-}$for $\alpha=1$ are drawn in different colors within the first Brillouin zone.

and 1 along the $q$-path, $\Gamma-\mathrm{X}-\mathrm{M}-\mathrm{X}^{\prime}-\Gamma$ are drawn in different colors (Fig. 3). Dispersion 
relations $E_{1}^{+}$and $E_{1}^{-}$are always symmetric to each other about the zero-energy. On the other hand, $E_{3}^{+}$and $E_{2}^{+}$are symmetric to $E_{2}^{-}$and $E_{3}^{-}$, respectively about the zero-energy only when $\alpha=0$. So, DOS is also symmetric about the zero-energy when $\alpha=0$. Band gap opens up where DOS vanishes. In this system, DOS is always non-zero leading to the appearance of no band gap in the TB band structure for any values of $\alpha$. No qualitative change in the band structure is observed when the amplitudes of hopping parameters across $\delta_{\mathbf{2}}$ and $\delta_{\mathbf{3}}$ bonds are made different from each other. Only the band-width gets extended when the hopping amplitude across the $\delta_{\mathbf{3}}$ bond is made larger than that of the $\delta_{\mathbf{2}}$ bond.

The appearance of sharp peak in DOS corresponds to the presence of VHS in the band structure. As a result, the nature of DOS in the TB band structure of this pentagonal lattice indicates the presence of VHSs at two different energy values. Appearance of similar pair of VHSs has been observed earlier in case of another 2D system, graphene [13]. Both positions and heights of VHS-peaks vary with $\alpha$. For $\alpha=0$, locations of VHS-pair are symmetric about the zero-energy in the band structure, which is again similar to the case of graphene. Heights of two VHS peaks are the same at this point too. Both the symmetries are lost as soon as $\alpha \neq 0$. With the increase of $\alpha$, separation between two VHSs gets reduced. Height of the upper VHS peak becomes shorter and it moves toward bottom with the increase of $\alpha$. On the other hand, height of the lower VHS peak increases with the increase of $\alpha$ and it shifts toward bottom when $0<\alpha<1 / 2$. But the opposite behavior is observed in the same lower VHS peak when $1 / 2<\alpha<1$. VHS could be detected and characterized with the help of scanning-tunneling, optical and Raman spectroscopies whenever it appears very close to the Fermi energy $\left(E_{\mathrm{F}}\right)$. In graphene, positions of VHSs are symmetric about $E_{\mathrm{F}}$ and they emerge at far away from $E_{\mathrm{F}}$, which make them very difficult to observe [13]. Relative positions of VHSs could be changed by twisting the graphene layers, which in turn alter the values of hopping parameters [12]. Observation of low-energy VHSs by using scanning tunneling spectroscopy in the twisted graphene layers has been reported in the article [14]. Similarly, in case of prismatic pentagonal lattice, positions as well as heights of VHS-peaks could be varied by controlling the value of $\alpha$. VHS largely affects the optical and electrical properties of the materials. For example, optical absorption spectra of the twisted bilayer graphene are found to exhibit multiple peaks, which corresponds to the presence of multiple VHSs in its band structure [15]. However, in two-dimensional systems, electronic instabilities occur when VHS appears in the vicinity of $E_{\mathrm{F}}$ leading to the emergence of several phases of 
matter like superconductivity [16], ferromagnetism [17] and charge density waves [18].

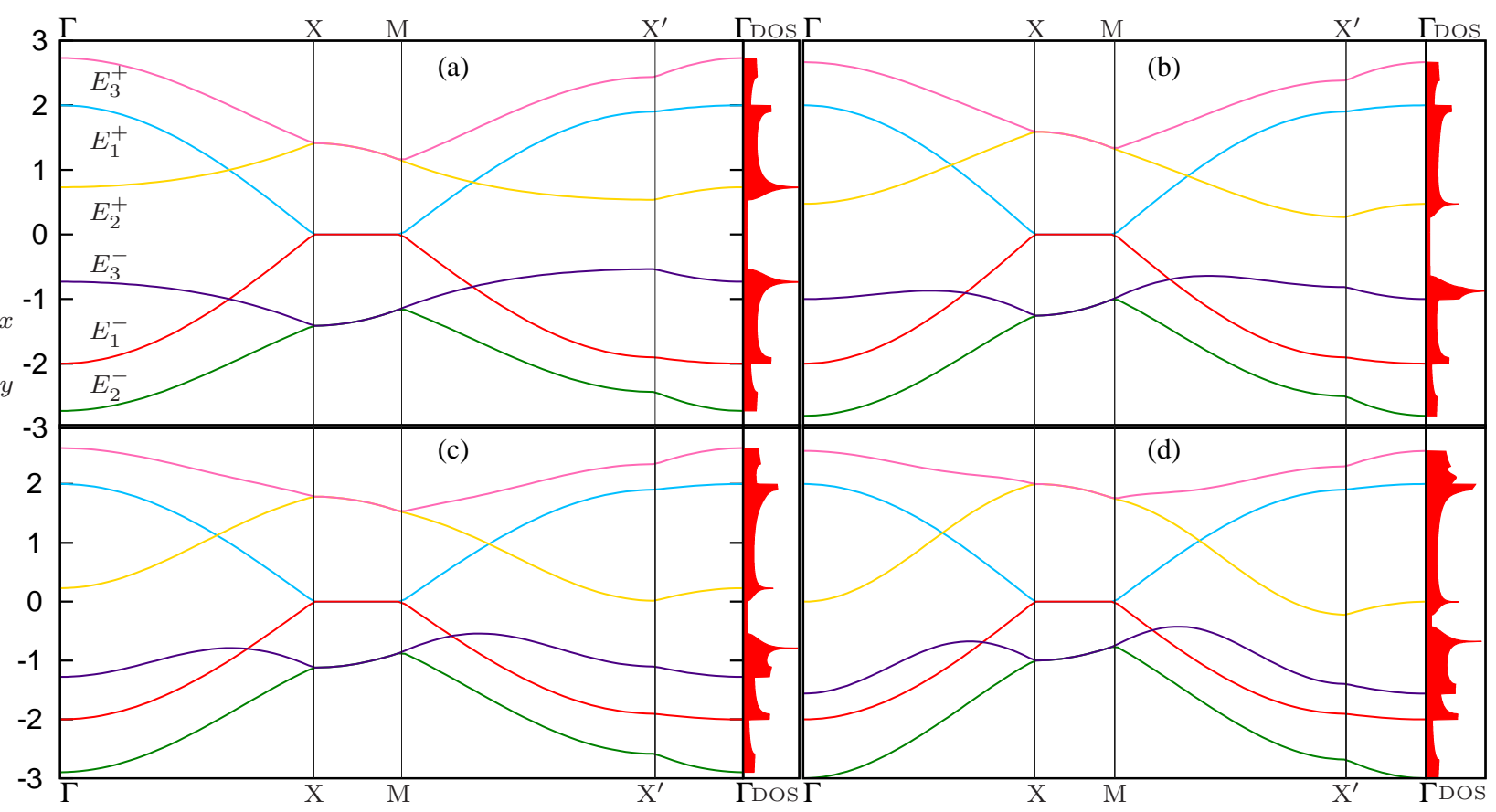

FIG. 3: (Color online) Dispersion relations, $E_{1}^{+}, E_{2}^{+}, E_{3}^{+}, E_{1}^{-}, E_{2}^{-}$and $E_{3}^{-}$and DOS for $\alpha=0$ (a), $\alpha=1 / 3$, Harrison point, (b), $\alpha=2 / 3$ (c) and $\alpha=1$ (d) along $q$-path $\Gamma-\mathrm{X}-\mathrm{M}-\mathrm{X}^{\prime}-\Gamma$.

\section{CONCLUSION}

In this study, we have clearly predicted the existence of no band-gap in the TB analysis on the prismatic pentagon lattice in contrast to the previous observation of a single band-gap for the Cairo pentagonal lattice. In addition, a pair of VHSs appears in the band structure at two different energies. Similar feature is observed before in graphene. Both positions and heights of the VHS-peaks are found to vary with the values of $\alpha$. Like the Cairo pentagonal lattice, no Dirac-cone is found. It would be noted that Dirac-cones are omnipresent in the 2D lattices with the coordination number of three, such as, honeycomb (graphene), 1/2-depleted (T-graphene) and 1/5-depleted square lattices [19]. However, in these pentagonal lattices coordination numbers are either three or four depending on the position of lattice sites. Recently, innovation of another class of 2D materials, such as pgraphene [1] and heptagraphene [20], resembling the graphene structure are gaining interests in which the basic structure of those lattices are generated by using the irregular pentagons 
and heptagons, respectively. This trend is due to the appreciation of exotic electronic properties observed in graphene. Although, p-graphene is made of only carbon atoms, the unit cell of heptagraphene is composed of ten carbon and four hydrogen atoms. Again, triggered by the success of graphene physics, realization of new carbon allotropes are gaining

considerable impetus. Possibility of stable p-graphene based on the Cairo pentagon may be realized very soon. Therefore, it is expected that another stable p-graphene based on the prismatic pentagonal lattice would be materialized in near future. Apart from the pure Cairo and prismatic pentagonal lattices, a class of 2D lattice structures made of several combinations of Cairo and prismatic pentagones could be generated which might give rise to novel electronic properties as well.

\section{ACKNOWLEDGEMENTS}

Authors are grateful to Prof. Indrani Bose for bringing the pentagonal lattice model to their notice. AKG acknowledges a BRNS-sanctioned research project, no. $37(3) / 14 / 16 / 2015$, India.

[1] S. Zhang et. al., Proc. Natl. Acad. Sci. USA 112, 2372-2377 (2015).

[2] M. Yagmurcukardes et. al., J. App. Phys. 118, 104303 (2015).

[3] T. Stauber, J. I. Beltran and J. Schliemann, Sci. Rep. 6, 22672 (2016).

[4] E. Ressouche et. al., Phys. Rev. Lett. 103, 267204 (2009).

[5] A. M. Abakumov et. al., Phys. Rev. B 87, 139902 (2013).

[6] H. Yamaguchi et. al., Sci. Rep. 5, 15327 (2015).

[7] I. Rousochatzakis, A. M. Läuchli and R. Moessner, Phys. Rev. B 85, 104415 (2012).

[8] M. Isoda, H. Nakano and T. Sakai, J. Phys. Soc. Jpn. 83, 084710 (2014).

[9] H. Nakano, M. Isoda and T. Sakai, J. Phys. Soc. Jpn. 83, 053702 (2014).

[10] M. H. Waldor, W. F. Wolf and J. Zittartz, Phys. Lett. 106A, 261, (1984); Z. Phys. B Condens. Matter 59, 43 (1985).

[11] U. Bhaumik and I. Bose, Phys. Rev. B 58, 73 (1998).

[12] W. A. Harrison, Elementary Electronic Structure (World Scientific, Singapore, 1999), ISBN 
981-023895-9.

[13] A. H. Castro Neto et. al., Rev. Mod. Phys. 81, 109 (2009).

[14] G. Li et. al., Nat. Phys. 6, 109 (2010).

[15] P. Moon and M. Koshino, Phys. Rev. B 87, 205404 (2013).

[16] W. Kohn and J. M. Luttinger, Phys. Rev. Lett. 15, 524 (1965).

[17] M. Fleck, A. M. Oleś and L. Hedin, Phys. Rev. B 56, 3159 (1997).

[18] T. M. Rice and G. K. Scott, Phys. Rev. Lett. 35, 120 (1975).

[19] Y. Liu et. al., Phys. Rev. Lett. 108, 225505 (2012).

[20] A. Lopez-Bezanilla, I. Martin and P. B. Littlewood Sci. Rep. 6, 33220 (2016). 